\title{
Mesial temporal lobe epilepsy with hippocampal sclerosis in two pairs of identical twins
}

\author{
Karatas $\mathrm{H}^{1}$, Dericioglu $\mathrm{N}^{1}$, Saygi $\mathrm{S}^{2}$ \\ Institute of Neurological Sciences and Psychiatry, Hacettepe University, Ankara, Turkey. \\ hulyak@hacettepe.edu.tr
}

\begin{abstract}
The most common pathology in adult patients with temporal lobe epilepsy is hippocampal sclerosis (HS), the etiology of which is not clear. There is a conflicting evidence in literature regarding whether HS is genetic or acquired. Twin studies can help to clarify the mechanisms of HS, but limited numbers of twins have been studied. We describe two monozygotic pairs, in whom the affected twin had mesial temporal lobe epilepsy and HS. The unaffected twin remained seizure free on long-term follow-up. HS was confirmed pathologically in one of the affected twins. Our data and other limited twin studies indicate that HS occurs as a consequence of prolonged repeated seizures or other events during childhood. In other words, some acquired factors may be more important than genetic ones in the etiology of HS (Fig. 2, Ref. 16). Full Text in PDF www.elis.sk.

Key words: hippocampal sclerosis, twin, mesial temporal lobe epilepsy.
\end{abstract}

The most common pathology in adult patients with temporal lobe epilepsy (TLE) is hippocampal sclerosis (HS) (1). It can be diagnosed using a brain MRI, and its resection is associated with an excellent seizure outcome (2). The etiology of HS is not clear. There is a conflicting evidence in literature regarding whether HS is genetic or acquired. Retrospective surgical studies showed that HS is associated with clinical histories of a prior initial precipitating injury (IPI) or medical event (3). Preliminary studies examining the hippocampi from children with severe seizures indicate that the immature hippocampus shows signs of seizure related injury (4). Other suggestions for the origin of HS include that it is part of a developmental abnormality or perinatal trauma or a consequence of multiple seizures (5-7). In addition, the growing numbers of genetic studies disclose the role of inheritance in many epilepsy syndromes.

Twin studies can help to clarify the origin of HS. So far, only two papers have reported monozygotic twin pairs, in whom only one of the twins had mesial TLE and HS, whereas the other twin was unaffected $(8,9)$. Both reports have suggested that HS is an acquired lesion secondary to prolonged seizures in early childhood.

Here we present two sets of identical twins, while only one of the twins suffered from mesial TLE with HS. Their follow-up

${ }^{1}$ Institute of Neurological Sciences and Psychiatry, Hacettepe University, Ankara, Turkey, and ${ }^{2}$ Department of Neurology, Faculty of Medicine, Hacettepe University, Ankara, Turkey

Address for correspondence: $\mathrm{H}$. Karatas, MD, Hacettepe University Institute of Neurological Sciences and Psychiatry, 06100 Sihhiye, Ankara, Turkey.

Phone: +90.312.3052620, Fax: +90.312.3093451

Acknowledgements: We confirm that we have read the Journal's position on issues involved in ethical publication and affirm that this report is consistent with those guidelines.

None of the authors has any conflict of interest to disclose. was long enough ( $>10$ years), to allow us to be confident about the unaffected twin.

\section{Patients}

Pair 1: The parents were not consanguineous. The pregnancy was normal; the birth was premature (at 30 weeks). These female twins were born by normal vaginal delivery. There was no difference in birth weight between them, suggesting that the intrauterine environment was similar for both. The affected twin was the firstborn. They did not have febrile seizures, a history of cerebral infection, or head trauma as predisposing factors. Twin zygosity was determined by a validated questionnaire and clinical examination.

Twin 1A (affected): A 17-year-old woman was admitted to our clinic for drug resistant complex partial seizures recurring 10 times/month since 11 years of age. There was no history of status epilepticus. Her neurological examination was normal. She was admitted to long-term video-EEG monitorisation unit for five days and four habitual seizures were recorded. The interictal EEG revealed spike or sharp waves in the right temporal lobe. Epigastric rising sensation was reported as an aura. During the seizures, right hand automatisms and contralateral dystonic posturing with unresponsiveness were observed. The ictal EEG revealed a buildup of typical rhythmic sharp theta activity in the right temporal lobe. Her cranial MRI disclosed right hippocampal atrophy and hyperintensity on FLAIR and $\mathrm{T}_{2}$ weighted images, concordant with HS (Fig. 1). She had a right temporal resectomy with amygdalohippocampectomy at the age of 20, after a detailed presurgical work-up including ictal EEG, WADA test, and neuropsychological testing. Her neuropathological assessment confirmed HS. She has been seizure free for 11 years during the postoperative follow-up.

Twin 1B (unaffected): There was no history of febrile or afebrile seizures. She was completely healthy and seizure-free during 

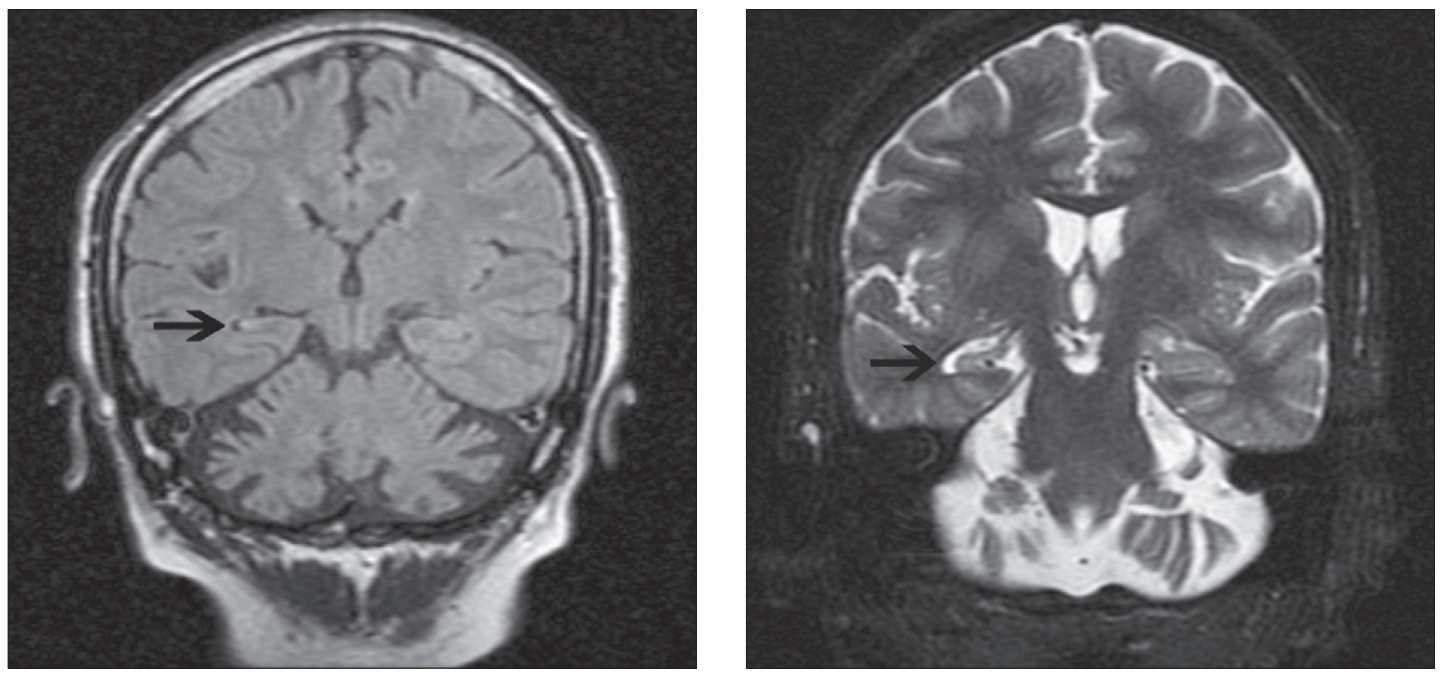

Fig. 1. Cranial MRI of twin $1 \mathrm{~A}$ showed right hippocampal atrophy in $\mathrm{T} 1$ weighted images and hyperintensity in $\mathrm{T} 2$ weighted images concordant with hippocampal sclerosis.
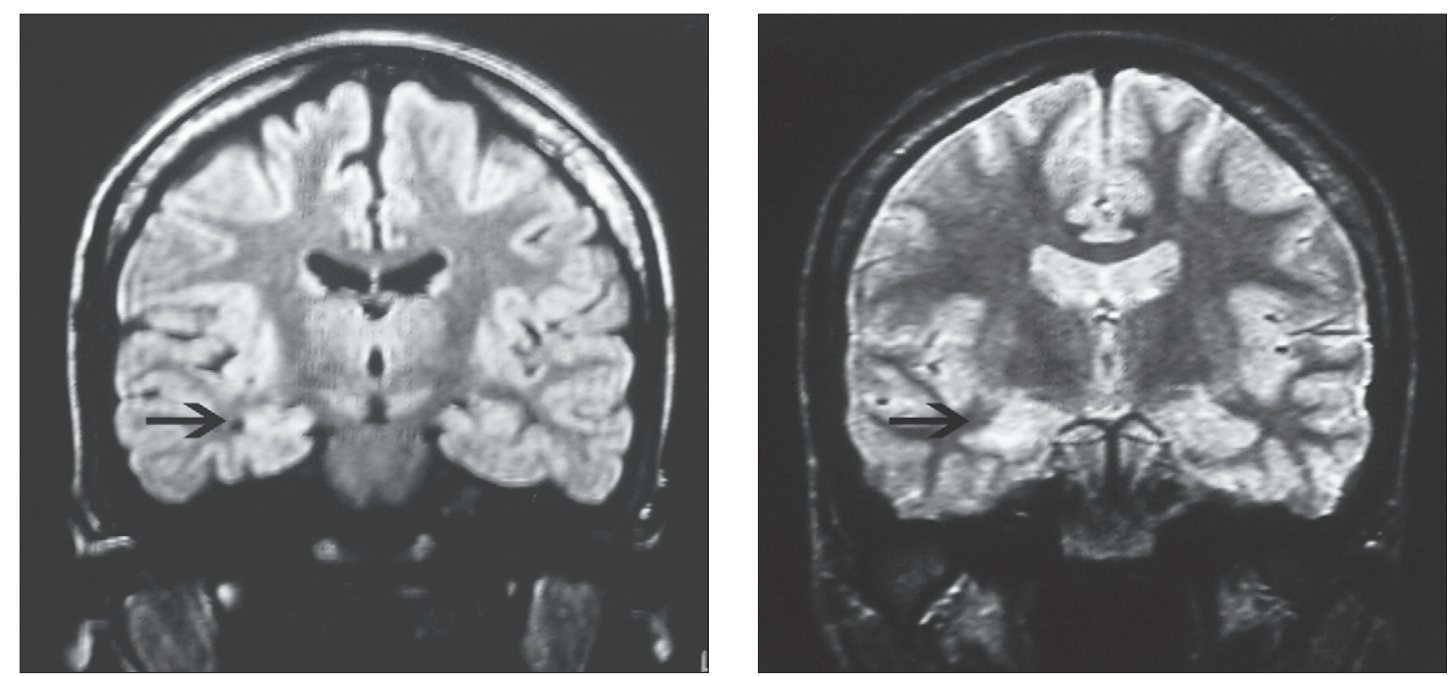

Fig. 2. Cranial MRI of twin 1B showed right hippocampal atrophy in $\mathrm{T} 1$ weighted images and hyperintensity in $\mathrm{T} 2$ weighted images concordant with hippocampal sclerosis.

the 14-year follow-up of her sister. Brain MRI could not be performed because the family did not have social security insurance and could not afford to pay for the procedure.

Pair 2: There was no consanguinity between the parents. The pregnancy was normal and birth was at 40 weeks. These male twins were born by normal vaginal delivery. There was no difference between their birth weights. The affected twin was the first-born. There was no history of perinatal injury. They did not have febrile seizures, cerebral infection, or head trauma. Twin zygosity was determined by a validated questionnaire and clinical examination.

Twin 2A (affected): He was admitted to our clinic at age 18, due to seizures. His seizures started at the age of 30 months and were described as "drowsiness" by the parents. For three years, his seizures recurred at a frequency of once per month. He remained seizure free between 6 and 17. At the age of 18, second- ary generalized tonic clonic seizures had started. He had been on carbamazepine monotherapy. His physical and neurological examinations were normal except for minimal mental retardation. Interictal EEG showed right centrotemporal spike activity. Cranial MRI revealed right HS (Fig. 2). He is still having seizures despite antiepileptic therapy but these have been rare, once a year during the 10 years of follow-up.

Twin 2B (unaffected): There was no history of seizures. He was perfectly well except for rare migraine-like headache attacks. There was no pathology in his cranial MRI.

\section{Discussion}

Our findings in two pairs of monozygotic twins suggest that mesial TLE due to HS may more likely have an acquired basis 
rather than genetic causes. This is in agreement with two previous papers where one (9) and three (8) identical twin pairs, discordant for the presence of mesial TLE and HS were reported. In the previous publications, the affected twins had prolonged febrile convulsions prior to the development of epilepsy, leading to the conclusion that HS is an acquired lesion secondary to prolonged seizures in early childhood. Our findings however do not support this result, since neither of the affected twins had a history of prolonged febrile convulsion. In fact, we were not able to reveal any predisposing risk factors for epilepsy such as perinatal insult, birth trauma, head injury, central nervous system infection, etc. Interestingly both of the affected twins were the first born; nevertheless this has not been recognized as a risk factor by Jackson et al (8).

Familial mesial TLE cases suggest that mesial TLE with HS may have a genetic basis. The MRI study of two families with TLE and febrile convulsion in some individuals reported a unilateral hippocampal asymmetry even in some of the members without febrile convulsion, suggesting a genetic basis for HS (10). In the study by Kobayashi et al, the successive association of febrile seizures and MTLE or HS was not always observed in families with mesial TLE, some patients had HS without FS and other individuals had HS although they were asymptomatic (11).

Monozygotic twin studies seem to be a wonderful tool to study the genetic nature of diseases. Previously, Briellmann et al have analyzed 12 monozygotic discordant twins for factors explaining discordance (12). In 10 of these 12 pairs a clinical or MRI correlate of epilepsy was found. The two pairs with HS had previously been described by Jackson et al (8). Other pairs had vascular lesions, malformations of cortical development, etc. The authors concluded that occult acquired factors, such as prenatal insults, or genetic abnormalities resulting from postfertilization genetic processes could account for the discrepancy. It has been demonstrated previously that monozygotic twins need not share absolutely identical genomes (13), probably resulting from postfertilization factors. Therefore, the identical twins may not rule out genetic differences between the siblings.

Another possibility is the effect of a subtle acquired abnormality, either pre- or post-natal. As far as pre-natal factors are concerned, it has been suggested that fetal developmental abnormalities may be induced by transient circulatory instability in identical twins due to fetal-fetal transfusions. It has been hypothesized by Brodtkorb et al that "a proportion of cases with malformations of cortical development with obscure cause is the result of a "vanishing twin" causing transient hemodynamic disturbances in the survivor (14).

Various post-natal factors are well known in the etiology of mesial TLE. A history of febrile seizures in approximately half of the patients with severe TLE and HS was first noted by Falconer and has been repeatedly confirmed, although only a minority of children with febrile seizures develops TLE $(1,7)$. Evidence from clinical studies of TLE cohorts and follow up studies of children with febrile seizures suggests that prolonged or complex febrile seizures are primarily associated with the subsequent development of TLE (7). Latent infections by herpes viruses may have some influence on cases of HS with mesial temporal lobe epilepsy, and the activity of the latent virus in patients with HS should be more extensively studied to establish its role in active infection (15). None of our twins had febrile seizures or a history of meningitis/encephalitis during childhood. History alone may not rule out the effect of an unrecognized infection, but since we have not tested our viral hypothesis we cannot further speculate on that. It has also been suggested that HS may be part of a developmental abnormality (5). Excess white matter neurons, minor dysplastic changes, and other developmental lesions have been noted in patients with HS (5). Comparison of MRI examinations between the affected and non-affected twin may allow subtle abnormalities to be identified (12). However, we did not find any developmental abnormality in either the affected (2A) or the unaffected (2B) twin, or any minor differences on cranial MRI. It is also possible that a hippocampal lesion of malformative origin may have been present before the seizures. A combination of inherent pre-existing abnormalities in the temporal lobe and precipitating events (e.g. prolonged febrile seizures), may eventually cause an epileptogenic lesion in the hippocampus, i.e. the "two-hit hypothesis" (16).

Screening the whole genome in identical twins would help to clarify the presence or absence of postfertilization genetic differences. Once genetic variation is ruled out, the etiology of HS would be an acquired factor, either pre- (which would be difficult to test) or post-natal. This report can at least partly address the possible etiology of HS. These two pairs of twins suggest that HS does not occur only due to obvious pre-/perinatal injuries or genetic factors, as far as clinical findings and patient history are concerned. As only one twin of the pairs was affected and their seizures started during childhood, the possible etiology of HS seems to be acquired factors such as recurrent seizures in childhood. This finding is in agreement with the conclusion of Mathern and colleagues who stated that there was a strong association between a childhood seizure and the finding of HS at surgery (4). Given the evidence of genetic and acquired causes for mesial TLE and HS in literature, it is likely that the cause is heterogeneous, and may even vary between families. However, we suggest that some acquired factors may be more important than genetic factors in the etiology of HS.

\section{References}

1. Falconer MA, Serafetinides EA, Corsellis JAN. Etiology and pathogenesis of temporal lobe epilepsy. Arch Neurol 1964; 10: 233-240.

2. Berkovic SF, McIntosh AM, Kalnins RM, Jackson GD, Fabinyi GC, Brazenor GA, Bladin PF, Hopper JL. Preoperative MRI predicts outcome of temporal lobectomy: an actuarial analysis. Neurology 1995; 45: 1358-1363.

3. Mathern GW, Pretorius JK, Babb TL. Influence of the type of initial precipitating injury and at what age it occurs on course and outcome in patients with temporal lobe seizures. J Neurosurg 1995 a; 82: 220-227.

4. Mathern GW, Babb TL, Vickrey BG, Melendez M, Pretorius JK. The clinical-pathogenic mechanisms of hippocampal neuron loss and surgical outcomes in temporal lobe epilepsy. Brain 1995 b; 118: 105-113. 
$441-444$

5. Raymond AA, Fish DR, Stevens JM, Cook MJ, Sisodiya SM, Shorvon SD. Association of hippocampal sclerosis with cortical dysgenesis in patients with epilepsy. Neurology 1994; 44: 1841-1845.

6. Berkovic SF, Howell RA, Hay DA, Hopper JL. Twin birth is not a risk factor for seizures. Neurology 1993; 43: 2515-2519.

7. Verity CM, Ross EM, Golding J. Outcome of childhood status epilepticus and lengthy febrile convulsions: findings of national cohort study. BMJ 1993; 307 (6898): 225-228.

8. Jackson GD, McIntosh AM, Briellmann RS, Berkovic SF. Hippocampal sclerosis studied in identical twins. Neurology 1998; 51: 78-84.

9. Schulz R, Ebner A. Prolonged febrile convulsions and mesial temporal lobe epilepsy in an identical twin. Neurology 2001; 57 (2): 318-320.

10. Fernández G, Effenberger O, Vinz B, Steinlein O, Elger CE, Döhring W, Heinze HJ. Hippocampal malformation as a cause of familial febrile convulsions and subsequent hippocampal sclerosis. Neurology1998; 50 (4): 909-917.

11. Kobayashi E, D’Agostino MD, Lopes-Cendes I, Berkovic SF, Li ML, Andermann E, Andermann F, Cendes F. Hippocampal atrophy and T2-weighted signal changes in familial mesial temporal lobe epilepsy. Neurology 2003; 60 (3): 405-409.
12. Briellmann RS, Jackson GD, Torn-Broers Y, Berkovic SF. Causes of epilepsies: insights from discordant monozygous twins. Ann Neurol 2001; 49 (1): 45-52.

13. Martin N, Boomsma D, Machin G. A twin-pronged attack on complex traits. Nat Genet 1997; 17 (4): 387-392.

14. Brodtkorb E, Myhr G, Gimse R. Is monochorionic twinning a risk factor for focal cortical dysgenesis? Acta Neurol Scand 2000; 102 (1): $53-59$.

15. Karatas H, Gurer G, Pinar A, Soylemezoglu F, Tezel GG, Hascelik G, Akalan N, Tuncer S, Ciger A, Saygi S. Investigation of HSV-1, HSV2, CMV, HHV-6 and HHV-8 DNA by real-time PCR in surgical resection materials of epilepsy patients with mesial temporal lobe sclerosis. J Neurol Sci 2008; 264 (1-2): 151-156.

16. Wieser HG; ILAE Commission on Neurosurgery of Epilepsy. ILAE Commission Report. Mesial temporal lobe epilepsy with hippocampal sclerosis. Epilepsia 2004; 45 (6): 695-714.

Received January 17, 2010. Accepted April 15, 2012. 